\title{
Analysis of Cultural Education and Behavior in Colleges and Universities Based on Image Recognition Technology
}

\author{
Nan Jia $\mathbb{D}^{1,2}$ and Huilan Jing ${ }^{1}$ \\ ${ }^{1}$ School of Marxism, Dalian University of Technology, Dalian, 116024 Liaoning, China \\ ${ }^{2}$ School of Marxism, Liaoning Institute of Science and Technology, Benxi, 117004 Liaoning, China \\ Correspondence should be addressed to Nan Jia; jiananmy@lnist.edu.cn
}

Received 5 October 2021; Revised 29 October 2021; Accepted 1 November 2021; Published 25 November 2021

Academic Editor: Xin Ning

Copyright (c) 2021 Nan Jia and Huilan Jing. This is an open access article distributed under the Creative Commons Attribution License, which permits unrestricted use, distribution, and reproduction in any medium, provided the original work is properly cited.

\begin{abstract}
When the rationality and importance of cultural education in colleges and universities have been confirmed, the key question is how to better realize cultural education. This requires an in-depth understanding and grasp of the cultural education work of colleges and universities, analyzing the internal logic contained in this education system and then discussing its practical rationale. This paper proposes a multiobjective Support Vector Machine (SVM) image recognition method for college cultural and educational behaviors based on three decision-making theories. Aimed at the problem of classification errors caused by image segmentation in image recognition of cultural and educational behaviors in colleges and universities, it is found that the reason that causes the same target to be divided into different subblocks is that the ordinary SVM classifier has only two choices of "yes" and "not" in classification. It is impossible to set the conditions for the classification of cultural and educational behavior images to satisfy all the existing conditions. In order to solve the above problems, this paper combines the three decision-making ideas with the image recognition of university cultural education behaviors and designs a multiobjective SVM image recognition method of university cultural education behaviors based on the three decision-making theories. We establish a higher education service quality structure model and use LISREL to explore the causal relationship among students' perceived service quality, university image, student's perceived service value, student trust, student satisfaction, and student behavior intentions in the field of higher education. The empirical results show that student satisfaction has a significant direct and positive effect on student behavior. Service quality has a significant direct and positive impact on perceived value and student trust. The perceived risk of higher education services determines the image of colleges and universities, and student trust plays an important role. The image of colleges and universities acts on student satisfaction through perceived value and student trust. The image of colleges and universities has a direct impact on students' behavioral inclination, and it acts on students' behavioral inclination through student trust and student satisfaction. Among the influences on behavior tendency, the direct positive effect of student trust is the largest, followed by the image of colleges and universities and the quality of service.
\end{abstract}

\section{Introduction}

With the general trend of artificial intelligence, its branch image recognition has also received close attention. As a collection of image processing and pattern recognition, it is a technology that uses computers to understand images to distinguish different target objects [1]. At present, image recognition is very common in daily life and business society, such as airport security check identity verification, commuting fingerprint check-in, or iris access control, and industry research is also very extensive, such as transportation, medical care, and agriculture. With the continuous development of the social economy and the advancement of the popularization of higher education, the employment pressure of undergraduates in our country is increasing, and "difficulty in obtaining employment" has become a common situation in society [2]. Under the impact of the social wave of the rise of high and new technology, the demand for structural talents in various enterprises is constantly expanding, and the demand for talents with strong professional skills is 
increasing. In order to increase the employment rate of graduates, colleges and universities have strengthened the training of students' professional skills to a certain extent. From a long-term perspective, professional education is important, but cultural quality education is also indispensable. It should run through the whole process of college students' training and education [3]. The educational goal of colleges and universities is to train people with all-round development of physical and mental health. Now, more emphasis is placed on national quality, cultural heritage, and patriotism. The core point of improving these is to strengthen the country's cultural quality education.

The cultural education system has been in operation in the university environment, but people's understanding of this way of education still has a certain degree of ambiguity [4]. For example, in previous studies, the understanding of cultural education includes campus culture education, environmental culture education, and subject culture education. There is even a view that all education is cultural education in the final analysis. Mentioning "culture" seems to be able to describe a lot without thinking, but the answer to "what the hell is" is dumb. People's understanding of cultural education is the same. It interprets the connotative characteristics of cultural education in colleges and universities and analyzes its inherent logic on this basis. These rational interpretations can enhance people's understanding of cultural education in colleges and universities. Education is the true appeal of ideological and political education, and cultural education is an important part of ideological and political education [5]. Under the conditions of cultural globalization and under the background of building a socialist cultural power, the study of cultural education is a modern development of traditional ideological and political education based on society and inculcation of knowledge and education. It is both an update of educational concepts. It is also the development of educational methods. System theory emphasizes the whole and connection. The cultural education system of colleges and universities is to summarize and integrate the elements of cultural education according to the scientific and logical relationship in accordance with the principles and methods of system theory, so as to play a role that exceeds the sum of the effects of individual elements and enhance the effectiveness of ideological and political work in colleges and universities [6].

This paper constructs a classifier for image recognition of cultural and educational behaviors in colleges and universities based on three decisions. This paper is aimed at the problem that the traditional image segmentation of cultural and educational behaviors in colleges and universities are all regular rectangles, and the same subobjectives may be divided into different submodules. Compared with the commonly used methods of image recognition of cultural and educational behaviors in colleges and universities, this paper designs and builds a college based on three decisions. The selection of judgment conditions is obtained by retraining the previous classification results. Through a limited number of iterations, the university cultural and educational behavior image segmentation divides the same target into the same submodule as much as possible, thereby solving the problem of errors in the image segmentation of the university cultural and educational behavior and making the university cultural and educational behavior image segmentation more accurate. This paper introduces the three decision-making theories into the traditional SVM image recognition of cultural and educational behaviors in colleges and universities, designs a classifier based on the concept of the three decision-making theories, and establishes a recognition model of cultural and educational behavior images in colleges and universities to improve the accuracy of the recognition of cultural and educational behavior images in colleges and universities. Through the analysis of the three decision-making theories, the concept of delayed decisionmaking is humanized in the field of cultural and educational behavior image recognition in colleges and universities, and the delayed decision-making part is iteratively processed, and finally more accurate recognition results are obtained. We develop an educational service quality scale to analyze the service quality in the field of higher education, establish a higher education service quality measurement model, and use mathematical statistics to derive the factors of higher education service quality attributes. Confirmatory factor analysis shows that the reliability and validity of the factors are high, and the second-order confirmatory factor analysis shows that they constitute higher-order factors of education service quality attributes. This article will introduce the advanced ideas of service marketing into the education quality management from the perspective of university education service quality management, adopt the research method of combining theoretical research and empirical research and qualitative analysis and quantitative analysis, and systematically analyze higher education.

\section{Related Work}

Image recognition is the process of detecting, separating, and cognizing target objects in simple or complex backgrounds. It is a basic behavior that is vital to the survival of animals. Human activities depend to a large extent on the classification or recognition of a large number of visual objects [7]. We can recognize these objects quickly and effortlessly even under different lighting conditions or when they are blocked by other objects in a complex visual environment. People form and continuously improve their own object classification principles and visual recognition systems through continuous learning of new objects. When the observer encounters a new object and does not have the conceptual information of the classified object in advance, the classification principle he formed before will serve as the basis for classifying the new object [8]. In the process of learning to recognize basic objects, humans can detect consistent features with minimal changes between individuals. These features represent most samples of an object class, thereby extracting class invariance and continuously improving their classification principles and recognition system [9].

The important reason for the long-term survival of human beings in nature is that they can quickly recognize and understand their environment and make corresponding response plans. The key is to use human's own visual system 
to locate and identify targets and at the same time realize visual scenes. If the computer can automatically recognize images, it will further enrich and facilitate human life. This makes image recognition technology one of the important research directions in the field of artificial intelligence and big data analysis. Image recognition refers to the use of computer vision, pattern recognition, machine learning, and other technical methods to automatically recognize the concept of one or more target semantics in the image, and the generalized image recognition also includes the concept recognition of image region positioning. Image recognition technology can meet the user's vision application needs in different scenarios, including Internet-based image retrieval and data mining, human-machine dialogue, and information services on smart terminals, such as mobile devices and smart robots.

There are different opinions on the construction of the cultural education system in academia, but most of them focus on the specific content, practical approach, and guarantee mechanism of the cultural education system [10]. Relevant scholars proposed that higher vocational colleges should start with the concept system, curriculum system, implementation system, and guarantee system to build a complete professional cultural education system [11]. Researchers believe that schools must first scientifically construct an ideal model of a practical-oriented regional excellent cultural education system, secondly creatively construct a practical model of a practical-oriented regional excellent cultural education system, and finally effectively construct a practical-oriented regional excellent cultural education [12]. The guarantee mechanism of the system can form a unique practice-oriented school regional cultural education system. Related scholars believe that one of the important connotations of the construction of the school cultural education system in the new era is to use the concepts of the new era to guide the construction of the school's cultural power, which mainly includes spiritual, material, behavioral, institutional, and other aspects [13]. Only when the main body of cultural power construction actively participates in the various cultural constructions of the school, a good development situation may emerge.

Relevant scholars pointed out that the understanding of cultural quality education should be viewed from three different levels [14]. The three levels have a progressive relationship, from shallow to deep, and cannot be viewed independently: colleges and universities should take "improving the cultural literacy of college students" as the first priority. Researchers believe that the humanities used in the cultivation of college students' cultural qualities should be based on the thinking and behavior of college students, focusing on social practice and urging college students to exert conscious initiative in promoting social development [15]. Relevant scholars pointed out that only relying on the means of imparting knowledge will never achieve the goal of cultural quality education [16]. It should be combined with the overall progress and development of people and society; that is, colleges and universities should not only pay attention to the transmission of knowledge but also pay attention to morality when cultivating talents [17]. For the organic combination of the cultivation and cultivation of sentiment, colleges and universities should carefully design courses and pay attention to the creation of a fine and harmonious environment. Relevant scholars believe that in cultural quality education, the role of people should be emphasized (people-oriented), and the cultivation of "full" people should also be emphasized at the same time as the cultivation of individuality [18]. Individuality is the source of innovation and creativity. Relevant scholars pointed out that the cultural quality education of college students should be combined with the improvement of teachers' cultural literacy, ideological and political, and focus on the comprehensive training of humanities and science of talents $[19,20]$.

\section{Practice Theory of Cultural Education in Colleges and Universities}

3.1. Operating Structure of Cultural Education in Universities. Cultural education is a kind of practical activity, which is related to people's epistemology, and is essentially an activity about people's cognition. The formation process of human cognition is the process of achieving the goals of subject and object on the basis of practice. It can be seen that the realization of the effect of cultural education also presents a structure of subject and object, which is the result of the integration of cultural and educational elements. The quality ecosystem of cultural quality education in colleges and universities is shown in Figure 1.

The cultural education system of colleges and universities depends on the social system, and it is also an organic operating system. Therefore, the grasp of its subject and object structure can be interpreted in the theory of system philosophy. In system philosophy, "subject" mainly includes three main forms: individual subject, group subject, and social subject. Regardless of the form of the subject, a basic feature is that it is social and conscious, and its activities are carried out with a certain purpose. "Object" mainly includes three object forms: natural object, social object, and spiritual object. The development of cognition activities is a process of mutual transformation between subject and object on the basis of practice, and the achievement of cognition goals is to realize the subjectivity of the object.

The subject of cultural education in colleges and universities should explore the content, source of motivation, and achievement of cultural education. In other words, one convenience for mastering the initiative and dominant force of this activity is the subject, while the party that is affected, received, and influenced is the object. According to the "differential order" structure of operation and development, teachers and students, schools, and cultural fields in society can all act as subjects, playing the roles of individuals, groups, and social subjects, respectively. But this does not mean that the subject of cultural education in colleges and universities has fallen into "relativism." What needs to be pointed out is that in the composition of these subjects, administrative forces, school environment, teachers, etc. still occupy important positions of the subject of cultural education in colleges and universities. Teachers and campus practitioners must not only play the main role of educating 

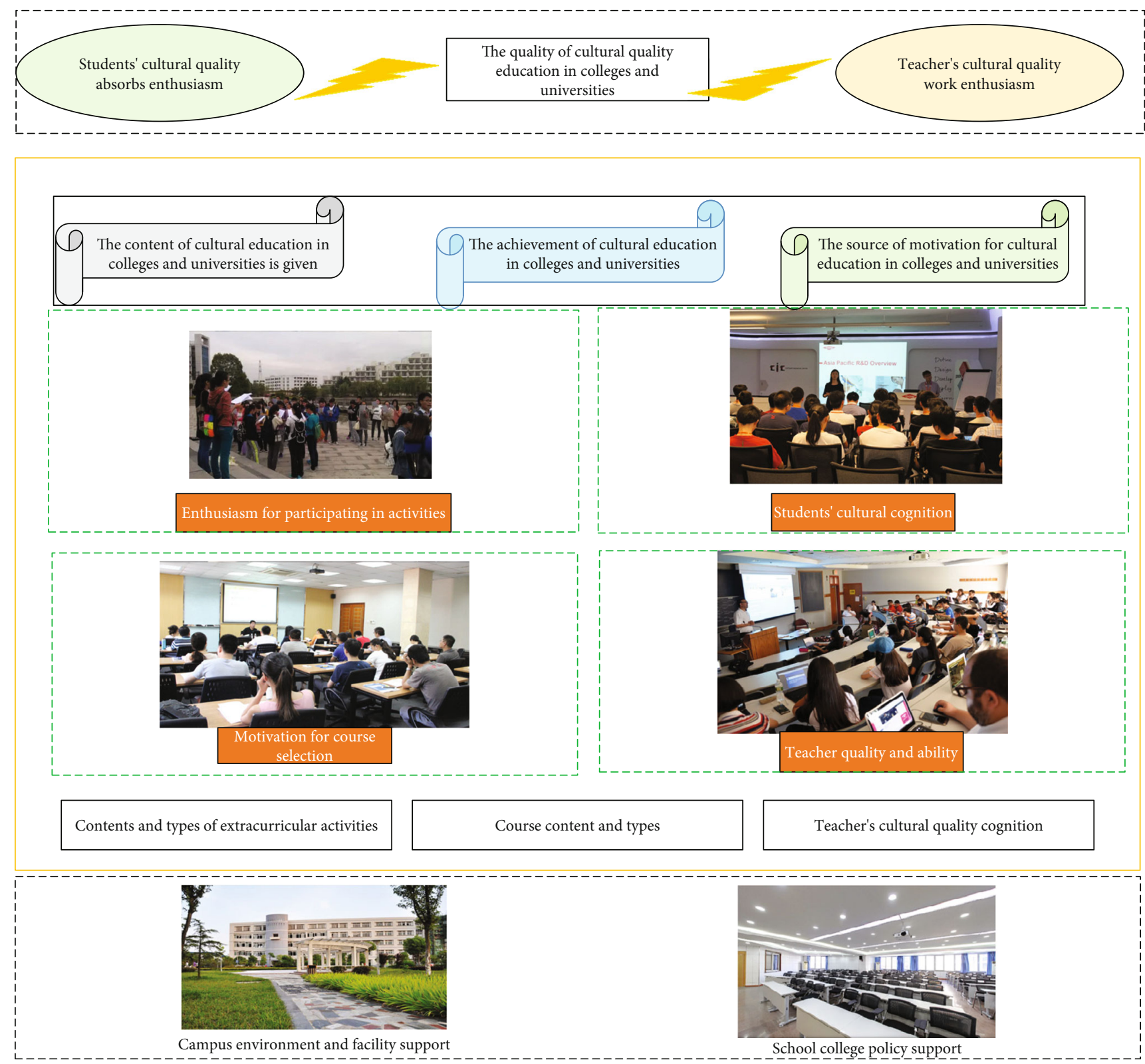

FIGURE 1: The quality ecosystem of cultural quality education in colleges and universities.

people, but in terms of the overall social environment and the "postmetaphor" role of students to teachers, they are also the objects of cultural education to some extent. Generally speaking, the student group is still an important part of the object of campus culture education.

\subsection{The Operating Mechanism of Cultural Education in} Colleges and Universities. When the object of education has certain cultural knowledge and forms a cognitive schema that conforms to a certain cultural content, the cultural education work has achieved a certain degree of success, but this is not the end, and it is not the complete completion of the cultural education work. The realization of the internalization of the cultural core at the level of educational objects is only half of the success of cultural education. It is important for the educational object to absorb and recognize the spiritual literacy reflected by this cultural core and enable such spiritual literacy to dominate daily behaviors. There are countless people who know and understand "integrity" in real life, while those who can truly achieve "integrity" in daily behavior will be greatly reduced. This involves the relationship between knowledge and action and the relationship between internalization and externalization. The goal of cultural education lies not only in simple knowledge transfer and transmission but also in the realization of the unity of knowledge and action in the object of education. The schematic diagram of the operating mechanism of cultural education in colleges and universities is shown in Figure 2.

According to the basic view of new behaviorism, the occurrence of behavior is dominated by conscious experience, and a behavior must have a corresponding behavioral motivation. Individual behavior is affected by factors such 


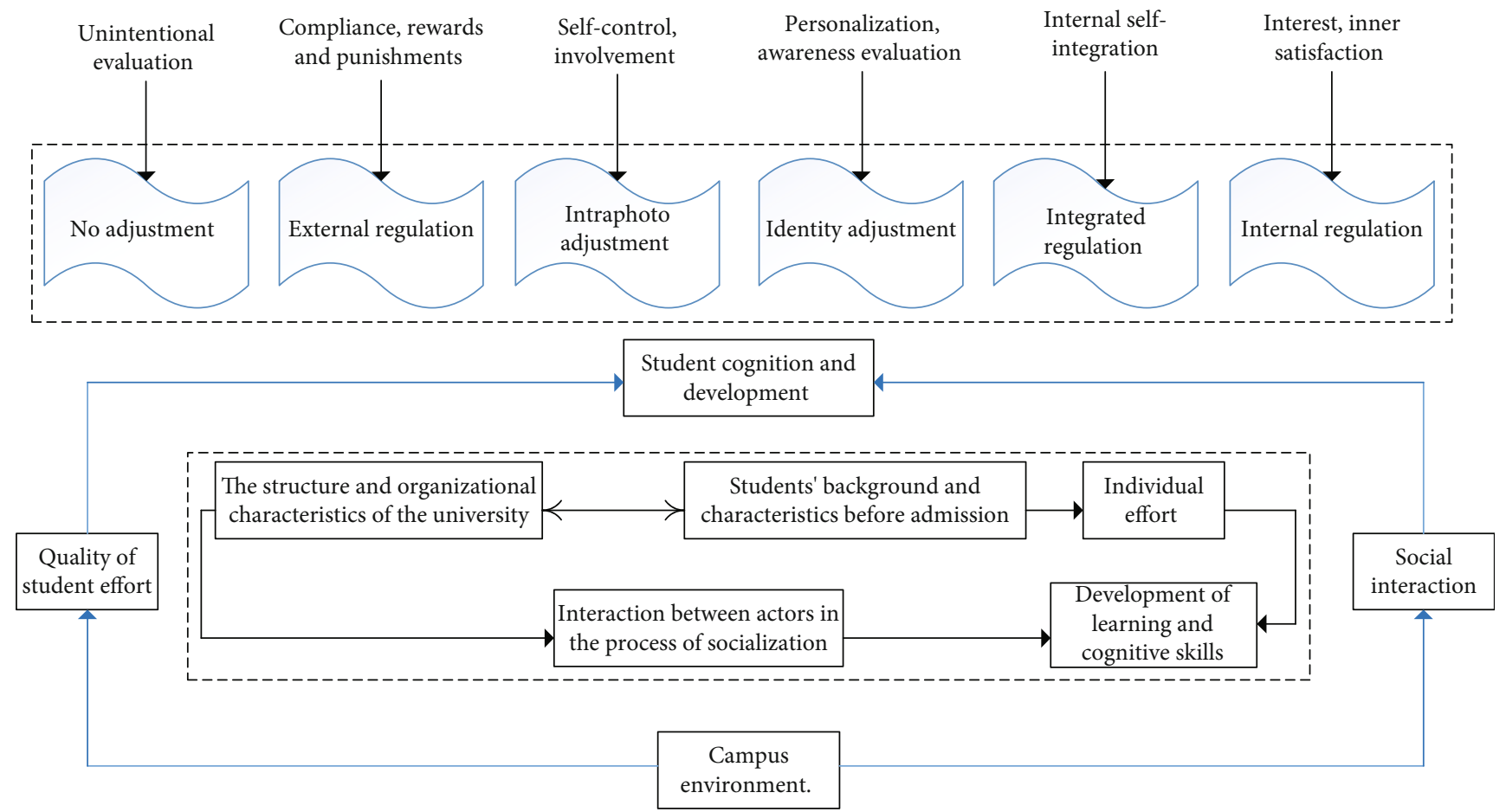

FIgURE 2: Schematic diagram of the operating mechanism of cultural education in colleges and universities.

as environment, experience, and age, and most of these variables are changed through learning. Therefore, in the process of a behavior, different stimuli and response variables will be formed through different stages of learning, which will have a decisive impact on individual behavior.

In contrast to the operation of the cultural education system in universities, its goal is to realize that the advanced socialist culture with Chinese characteristics has an impact on the ideological level of the main body of the university, so that the main body of the university has the content of the advanced socialist culture with Chinese characteristics. To achieve this goal, the first thing that needs to be solved is the conversion of the national macrotheory to the individual microideological level, that is, the assimilation and adaptation of the external cultural content discussed above to individual concepts. When the transformation of cultural content at the individual microlevel is completed, people will have new behavioral motives in their thoughts and concepts, and new variables that affect behavior will be produced. When similar thoughts exist in a certain range and function to produce similar behaviors, a group movement with common characteristics will be formed. This collective action has a strong social effect and can have an important leading role in social trends. In the university environment, if it is possible to realize that most of the behaviors of the main body of the university are dominated and guided by the advanced socialist culture with Chinese characteristics, it is the manifestation of the transformation of the effect of the university cultural education system from internalization to externalization. It is said that the cultural education system has achieved complete operation, and the effect of cultural education has finally been realized.

\subsection{Ways to Realize Cultural Education in Colleges and Universities}

3.3.1. Improve the System Design of Cultural Education. The cultural education of colleges and universities should give full play to the leading role of politics, continuously improve the system design, innovate the leading model, and establish a long-term mechanism for cultural education. In the process of overall development of colleges and universities, we must always adhere to the party's centralized and unified leadership of cultural education in colleges and universities, follow the basic rules of cultural education, and innovate and create education models and methods suitable for the college's own characteristics. Cultural education itself has a systematic and long-term nature, and the development of cultural education activities must give full play to the functions of various elements. There are rich organizational advantages in the university environment. It is necessary to give full play to the role of party organizations at all levels, league organizations, student associations, and other grassroots organizations, carry out various cultural education activities at all levels, and strengthen the theoretical study and core value education of college students. The cultural education of colleges and universities should be based on the main channel of curriculum teaching, grasp the key to teachers, establish and improve curriculum ideological- and politicalrelated systems, use curriculum teaching to enrich the campus cultural atmosphere, and realize cultural education.

3.3.2. Integrate the Educating Power of All Parties inside and outside the School. The realization of the effectiveness of cultural education in colleges and universities can not only rely 
on the "solo" of the school but also comprehensively coordinate the forces of all parties to form a cultural education "chorus." First of all, in terms of campus construction, we must pay attention to integrity and coordination. The creation of cultural atmosphere is not accomplished by setting up a functional department, but by infiltrating the concept of cultural education into all aspects of running a school. It is necessary to highlight cultural elements in the curriculum setting and campus environment construction, but also to create a cultural environment in the aspects of student accommodation, entertainment, and travel and cultivate teachers and students' patriotic and school-loving ideas with the ubiquitous and meticulous cultural infiltration concept. Secondly, it is necessary to make full use of external cultural education conditions such as social resources and family environment, link the construction of campus culture with the cultural construction of the city where it is located, and realize the organic integration of the cultural environment on campus and the cultural environment outside the campus.

\subsubsection{Create an Information Platform for Cultural} Education. The information age has provided more possibilities for the innovation of cultural education methods in colleges and universities. The rapid development and universal application of Internet technology are the biggest "trend" that colleges and universities must adapt to in ideological and political work. The emergence of emerging communication methods and information processing methods such as the Internet of things, blockchain, big data, and cloud computing has provided important technical support for colleges and universities to implement the task of fostering people and improve the quality of education. The cultural education work of colleges and universities should focus on the cultural education effect of cyberspace, which is concentrated in the two aspects of "establishing" and "breaking."

First of all, it is necessary to realize the "establishment" of network culture educating people. Colleges and universities should take an active and adaptive attitude to occupy the network position in time, make full use of Internet technology to build education platforms, enrich online content, and prevent the lack of mainstream cultural content in the network environment. The grasp of language and the guidance of topics are the key factors to realize the cultural education of the network environment. To carry out cultural education on the network platform, we must pay attention to the art of network language expression and be good at transforming profound theoretical content into popular network language to enhance the pertinence of cultural education.

Second, we must pay attention to the "breaking" of network content. In the network environment, all kinds of information are muddled down, and the good and bad are mixed, which often dissolves the education effect of mainstream culture. External forces and reactionary forces with ulterior motives often attract young students with weak discriminating ability under the guise of "recovering the truth" and "digging into the secret history." Therefore, another important way of educating people in online culture is to eradicate these weeds in cyberspace, seek to "stand" in "breaking," use true and authoritative information to elimi- nate students' doubts, and realize the implantation of positive and correct values.

\section{Multiobjective SVM Image Recognition Algorithm for University Cultural Education Behavior Based on Three Decision- Making Theories}

Three-branch decision-making is a model based on human understanding of decision-making. The three-branch decision-making thinking adds the concept of delayed decision-making to the traditional right and wrong decisionmaking. Through the delayed decision-making mechanism, the credibility of the decision-making is improved under the continuous addition of new decision-making conditions. The traditional image recognition technology of cultural education in colleges and universities is generally based on Support Vector Machine (SVM). Through the judgment of right and wrong, we classify the useful information of the cultural and educational behavior images of colleges and universities, so as to achieve the purpose of recognition of the cultural and educational behavior images of colleges and universities. The useful information of cultural and educational behavior images in colleges and universities mainly includes color, brightness, direction, contour, and other characteristics, and these characteristics are easily lost in the collection and transmission of cultural and educational behavior images in colleges and universities, so they will exist in the recognition process. Combining the three decision-making theories with the field of image recognition of cultural and educational behavior in colleges and universities can further judge and detect the fuzzy parts of the cultural and educational behavior images in colleges and universities by delaying decisionmaking and effectively train the determined parts. It effectively reduces the lack of useful information in the image of cultural and educational behavior in colleges and universities and improves the accuracy of image recognition of cultural and educational behavior in colleges and universities.

4.1. Multiobjective SVM Image Recognition of University Cultural Education Behavior. In this paper, the traditional recognition method is improved, and the efficient image segmentation method of university cultural education behavior is used, so that the university cultural education behavior image will not be divided into different subblocks because of the same target, thereby increasing the recognition rate. This paper integrates methods such as multifeatures, segmentation, detection, and multiclassifiers to conduct deep learning training on sample college cultural and educational behavior images. While reducing the loss of college cultural and educational behavior images, the target area is segmented and retrained. The composite SVM recognizer used in this paper is an improved version based on SVM recognition, which uses multiple methods such as fusion and segmentation to perform the final detection and recognition to improve the recognition rate of cultural and educational behavior images in colleges and universities. 
The traditional university cultural and educational behavior image recognition method is based on the composite SVM. We obtain the first hyperplane and the second hyperplane parallel to the hyperplane and the same distance from the sample data points. The hyperplane can be described by the following linear equation:

$$
g(x)=w^{T} x-b=0
$$

The first hyperplane is

$$
H_{1}: g_{1}(x)=w^{T}(-x)+b=-1 \text {. }
$$

The second hyperplane is

$$
H_{2}: g_{2}(x)=w^{T}(1-x)-b=1 \text {. }
$$

Among them, when $g(x)=0, x$ is a point on the hyperplane, vector $w$ is a vector perpendicular to the hyperplane $g(x)=0, w^{T}$ represents the transpose of the $w$ vector, and $b$ represents a constant. The sample data points of the tobe-identified college cultural and educational behavior image on the first hyperplane and the second hyperplane are the points closest to the separating hyperplane.

4.2. Three Decisions in Image Recognition of Cultural and Educational Behaviors in Colleges and Universities. Improving the accuracy of cultural and educational behavior image recognition in colleges and universities has always been a difficult point in the field of cultural and educational behavior image information in colleges and universities. The method of image recognition of cultural and educational behavior in colleges and universities is relatively single, and researchers are also easy to focus on the low-level visual feature points, which will lead to insufficient recognition of the amount of useful information in the cultural and educational behavior images in colleges and universities, thereby reducing the need for colleges and universities. This paper proposes a method of image recognition of cultural and educational behaviors in colleges and universities based on three decision-making methods, which makes use of the useful information in the cultural and educational behavior images of colleges and universities to a greater extent and makes the recognition of cultural and educational behavior images in colleges and universities more accurate. The understanding is that the content of the semantic information of the cultural and educational behavior images in colleges and universities is far more than the visual characteristics of the cultural and educational behavior images in colleges and universities. The current image segmentation of cultural and educational behaviors in colleges and universities usually directly divides the cultural and educational behavior images of colleges and universities into regular rectangles, which will cause the same target to be segmented into different subblocks of the cultural and educational behavior images of colleges and universities. The subblocks of cultural and educational behavior images are divided into different regions, which affects the accuracy of the recognition of cultural and educational behavior images in colleges and universities. The existing recognition methods improve the fusion of classifiers to improve the recognition performance of cultural and educational behavior images in colleges and universities. Due to the complexity of the content of cultural and educational behavior images in colleges and universities, the classification task of cultural and educational behavior images in colleges and universities is very difficult. The semantic classification of cultural and educational behavior images in colleges and universities is still challenging in the fields of college cultural and educational behavior image recognition, computer vision, and cognitive science.

In response to the above problems, this article proposes a three-branch decision-based image recognition method for cultural and educational behaviors in colleges and universities. The method uses the three-branch decision-making mechanism to process fuzzy information, thereby reducing the possibility of not being recognized because of fuzzy feature information. Since this method is to segment the college cultural and educational behavior images by continuously increasing the judgment conditions of the classifier, the college cultural and educational behavior images in different subblocks will be divided into the same subblock in the continuous iterative process. Each time the three decisionmaking classifiers add judgment conditions after filtering the existing conditions, the information added can be adjusted according to the current university cultural and educational behavior image's own attributes, and then the segmentation area can be adjusted continuously.

4.3. Design of a Multiobjective SVM Image Recognition Classifier for College Cultural and Educational Behaviors Based on Three Decisions. According to the characteristics of human cognition, three decision-making methods are adopted. Due to the unique delayed decision-making characteristics of the three decision-making, new decision information is constantly added to the initial decision conditions for decision-making condition judgments, so the classifier will take the divided positive and negative regions as new training after each classification is completed. The set is retrained to form a new judgment condition and added to the delayed decision-making area, until the delayed decision-making area can no longer be divided. Finally, we perform image recognition of cultural education behaviors in colleges and universities. Each training will add new decision-making conditions to make the classification results of the three decisionmaking classifiers clearer, and the part that delays the decision-making is smaller and smaller, until a certain critical value is reached, so that the image recognition of cultural education in colleges and universities achieves the ideal result.

Corresponding to the three decisions, we use $\alpha, \beta$, and $\xi$ to represent acceptance, rejection, and noncommitment, respectively. Suppose the evaluation function is defined as $\operatorname{Pr}(X)$, and the risk function is $R(\Delta \mid x)$, where $\triangle$ represents the decision-making action on $x$. Based on the cost matrix, the following risk estimates can be obtained for the two states:

The acceptance risk is

$$
R(\alpha \mid x)=\lambda_{\alpha p} \bullet P_{\beta}(-X \mid[x])-\left(P_{\beta}(-X \mid[-x])-1\right) .
$$




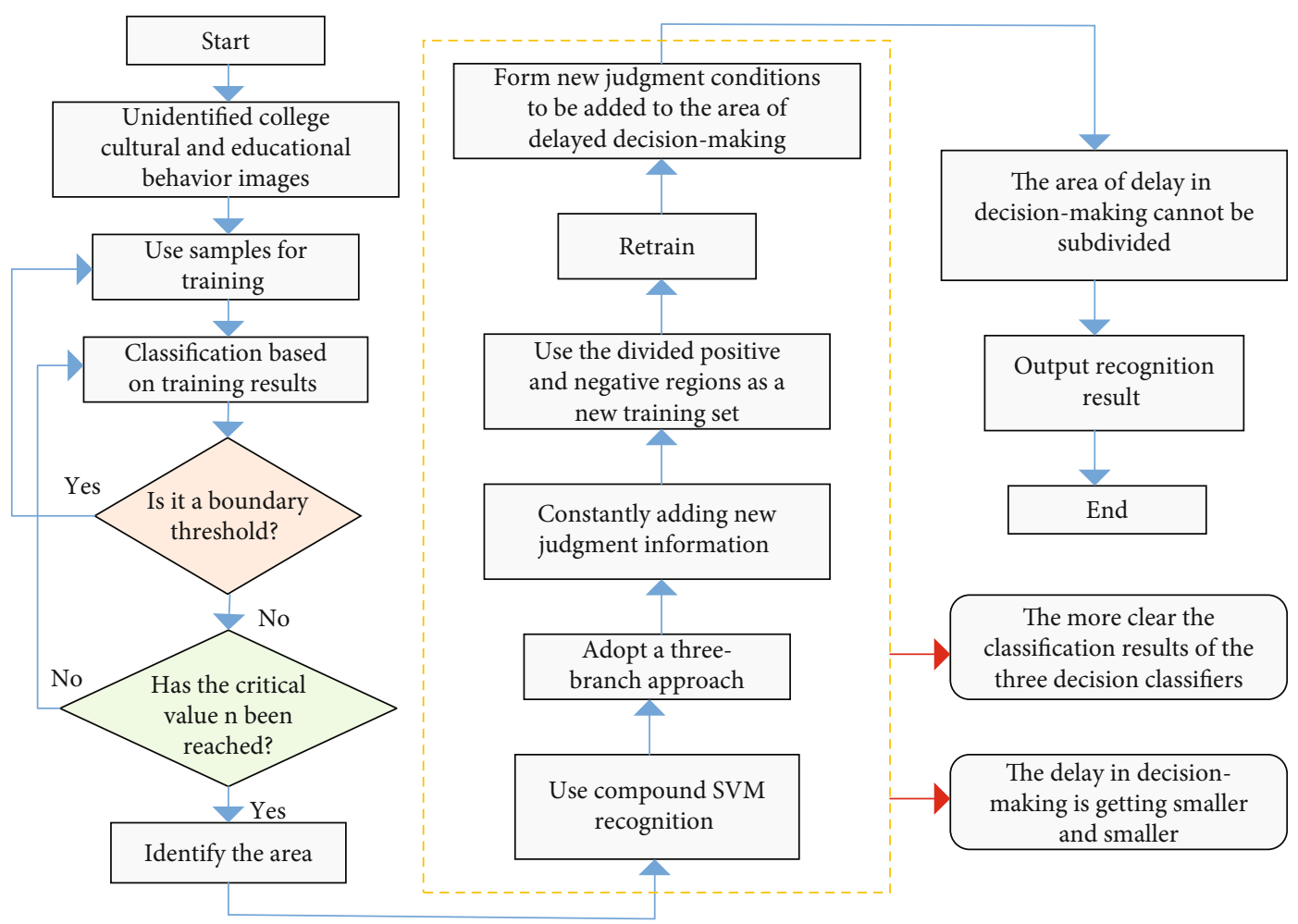

FIgURE 3: Flowchart of image recognition of cultural and educational behaviors in colleges and universities based on three decisions.

The rejection risk is

$$
R(\beta \mid x)=\left(1-\lambda_{\beta p}\right) \bullet P_{\beta}(X \mid[-x])-\left(P_{\beta}(-X \mid[-x])+1\right)
$$

The risk of noncommitment is

$$
R(\xi \mid x)=\left(1+\lambda_{\xi p}\right) \bullet P_{\beta}(X \mid[-x])+\left(P_{\beta}(-X \mid[-x])-1\right) .
$$

In the decision-making problem, based on the risk function, the decision action with the least risk is selected.

$\lambda \alpha p$ represents the cost of accepting decision-making when the acceptance conditions are met, $\lambda \alpha n$ represents the cost of accepting decisions that do not meet the decision-making conditions of the sample data set of college cultural and educational behavior images, and $\lambda \beta p$ represents the cost of accepting decisions that meet the decision-making conditions of the sample data set of college cultural and educational behavior images. $\Lambda \beta n$ represents the cost of rejecting decision-making under the condition of not satisfying the decision-making conditions of the sample data set of college cultural and educational behavior images, $\lambda \xi p$ represents the cost of noncommitting decisionmaking under the condition of satisfying the decisionmaking conditions of the sample data set of college cultural and educational behavior images, and $\lambda \xi n$ represents the cost of noncommitment decision-making under the decisionmaking conditions of the sample data set college cultural and educational behavior images, assuming that the risk function satisfies the following conditions:

$$
\frac{\lambda_{\xi p}-\lambda_{\beta p}}{\lambda_{\xi_{n}}+\lambda_{\alpha n}}<\frac{\lambda_{\xi_{n}}+\lambda_{\beta n}}{\lambda_{\xi p}-\lambda_{\beta p}} .
$$

Since the loss function satisfies the uncertainty condition of randomness, its mathematical mechanism is that the conditions for time occurrence are insufficient, so that there is no decisive causal relationship between the condition and the result. First, the event can be repeated under basically the same conditions; second, under basically the same conditions, an event may be manifested in multiple ways, and it cannot be determined in advance. In what specific way, third, all the possibilities of the event in various ways can be foreseen in advance (the probability of its occurrence in a certain way, that is, the probability of occurrence in the repeated process). The process of image recognition of cultural and educational behaviors in colleges and universities based on three decisions is shown in Figure 3.

\section{Empirical Analysis of Higher Education Service Quality}

\subsection{Analysis of the Attributes of Higher Education Service Quality}

5.1.1. Exploratory Factor Analysis. The 35 indicators can be classified into 7 factors, named curriculum-related, faculty services, academic resources, management mechanism, campus environment, school activities, and living and accommodation. The reliability coefficients of the seven factors reach 
TABLE 1: Reliability of service quality attribute factors.

\begin{tabular}{|c|c|c|}
\hline Dimension & Description & Reliability \\
\hline Course related & $\begin{array}{l}\text { Adequate teaching materials, teaching equipment, professional curriculum settings, } \\
\text { teaching content, and modernization of teaching equipment }\end{array}$ & 0.89 \\
\hline Faculty and staff services & $\begin{array}{l}\text { Faculty and staff are trustworthy, faculty and staff are willing to help, faculty and } \\
\text { staff are polite to others, faculty service efficiency, faculty and } \\
\text { staff business level, faculty clothing }\end{array}$ & 0.83 \\
\hline Academic resources & Library facilities, library resources, computer facilities, network & 0.82 \\
\hline Management mechanism & $\begin{array}{l}\text { Student participation, teacher-student communication, convenient service } \\
\text { time, feedback channels, service notifications, evaluation mechanism, } \\
\text { consideration of student interests, financial support }\end{array}$ & 0.87 \\
\hline Campus environment & Campus location, beautiful campus environment, campus safety & 0.79 \\
\hline School activities & Club activities, internship opportunities, student exchanges, sports activities & 0.77 \\
\hline Living and lodging & $\begin{array}{l}\text { Food quality, food prices, canteen facilities, accommodation conditions, } \\
\text { accommodation charges }\end{array}$ & 0.78 \\
\hline
\end{tabular}

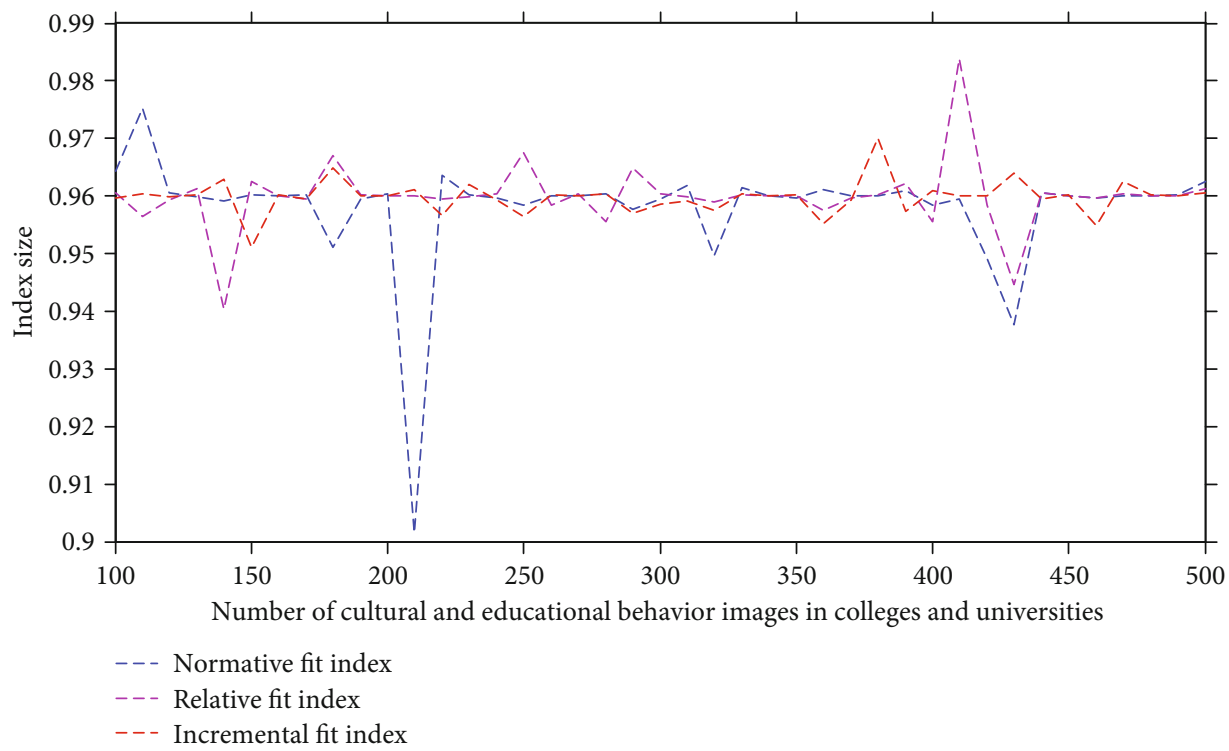

Figure 4: The goodness of fit of the second-order confirmatory factor analysis of service attributes.

$0.89,0.83,0.82,0.87,0.79,0.77$, and 0.78 , all exceeding the relevant recommended 0.6 , as shown in Table 1 .

5.1.2. Second-Order Factor Analysis. The confirmatory factor analysis results show that the curriculum, faculty services, academic resources, management mechanism, campus environment, school activities, and living and accommodation have a high degree of correlation among several variables. The approximate confidence interval test is conducted with standard errors. We use LISREL 8.7 to do a second-order factor analysis of the above variables. These variables can be classified as a higher-order factor "attribute of educational service quality."

The standardized estimates are all greater than 0.5 and less than the critical value of 0.95 , which is much greater than the 0.01 significance level of 2.58; that is, the factor load and $t$ value of all indicators of the evaluation model on their respective measurement concepts (latent variables) are rela- tively significant, indicating that the data is relatively significant. At the same time, no large errors occurred in the measurement errors of the observed variables, indicating that the next step can be an analysis of the overall suitability of the evaluation model.

Figure 4 shows the discriminant index results for evaluating the overall suitability of the model. The normative fit index (NFI), relative fit index (RFI), and incremental fit index (IFI) are all greater than 0.90, indicating that the model fits well.

The construction reliability and average variation extraction (second-order) are shown in Figure 5. The calculated reliability of the variable construction is between 0.72 and 0.78 , which is greater than the standard of 0.6 , indicating that the observed variable provides a credible construction measurement of the latent variable. The average variance variation extraction is between 0.72 and 0.80 , and both are greater than 0.5 . 


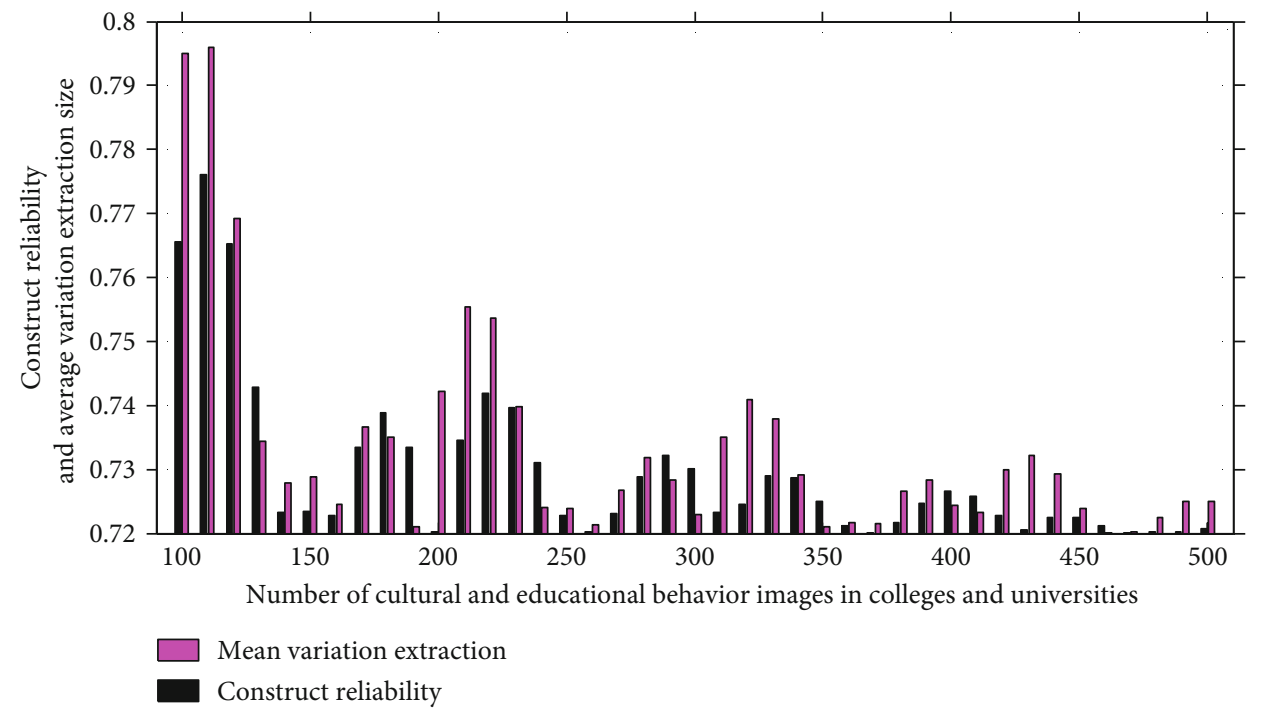

FIGURE 5: Construction reliability and average variation extraction (second-order).

\subsection{Analysis of the Relationship between the Qualities of Higher Education Services}

5.2.1. Data Description Analysis. Perceived curriculum is a combination of teaching materials, adequate teaching equipment, professional courses, modernization of teaching equipment, and teaching content. Perception of academic resources is a combination of library facilities, library resources, computer facilities, and the Internet; perception management mechanism is based on the convenience of service time, feedback channels, and student participation. Perceived campus environment is a combination of campus location, campus beauty, and campus safety; perceived school activities are community activities, internship opportunities, student exchanges, and physical activities; perceived life and accommodation are a combination of food quality, canteen facilities, food prices, accommodation conditions, and accommodation charges.

Cronbach's $\alpha$ of all concepts included in this study is higher than the minimum critical value of 0.6 recommended by related researches, indicating that the measurement model exhibits good internal consistency. The weight of each latent variable is shown in Figure 6.

5.2.2. Structural Equation Model Analysis. In the model designed in this article, expectation variables are not added. The formation of expectation is largely derived from the students' past consumption experience. Higher education is different from general services. In the field of education, students have little or no prior knowledge of the education to be received. Before consuming education services, although students' information sources include admission promotion, teachers, family members, or relatives and friends, the students themselves have no previous consumer experience to refer to, and they know very little about the level of services that the school will provide. Student expectations lack effectiveness, so student expectations are not included in the research model in this study.

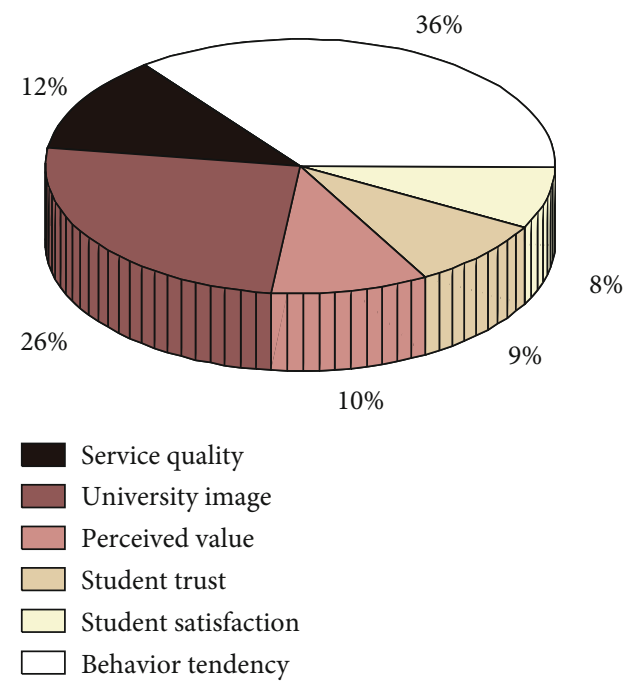

Figure 6: The weight of each latent variable.

The standardized coefficient value of the research sample is greater than 0.5 and does not exceed 0.95; except for the path from service quality to student satisfaction, the $t$ value is greater than 1.96 at the 0.05 significant level. Both the load and the $t$ value are more significant, indicating that the data has higher convergence effectiveness. The measurement error of the observed variable shows no large standard error and no negative error variance. These results show that there is no violation of the estimation phenomenon between the sample data of the measurement model, so the fitness of the overall model can be tested.

The construction reliability of each latent variable is greater than the 0.6 standard, indicating that the observation variable provides a credible construction measurement for the six latent variables, and the average variance variation extraction is greater than the 0.5 standard. Therefore, the reliability of each latent variable is relatively high. The comparison of the 


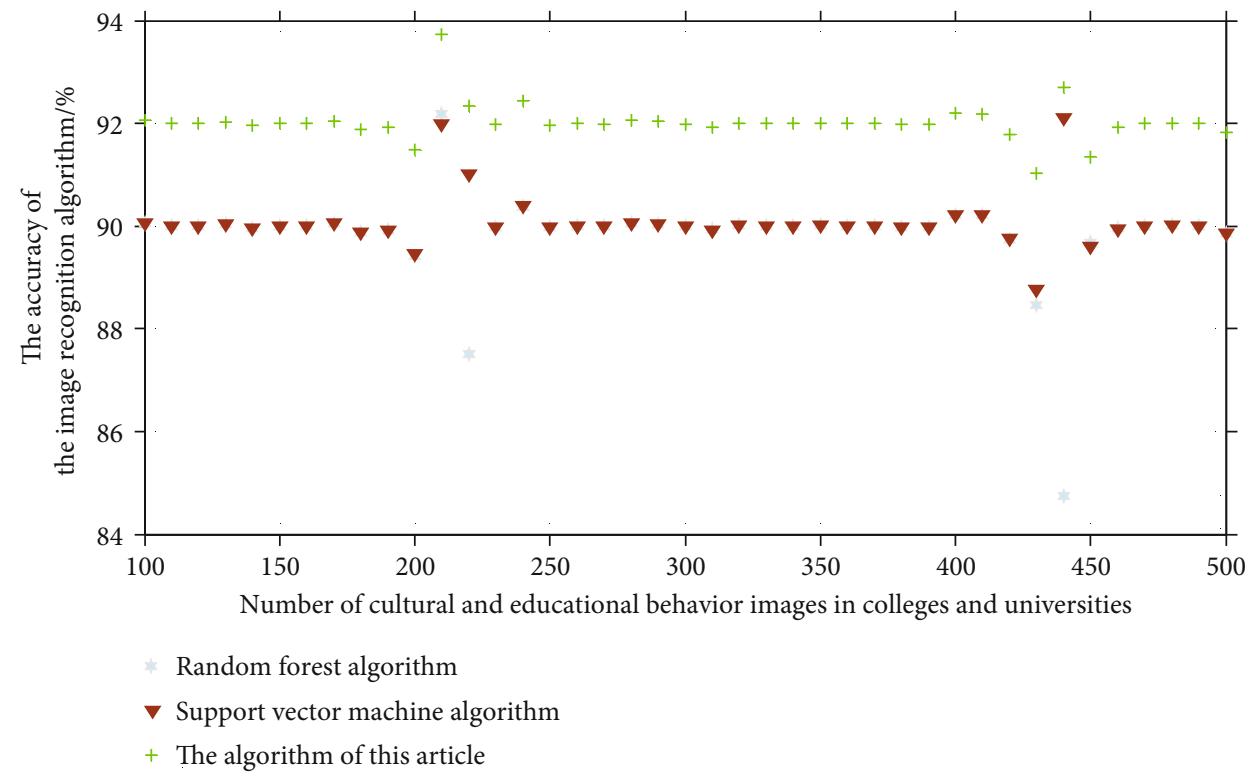

FIgURE 7: Comparison of accuracy of different image recognition algorithms.

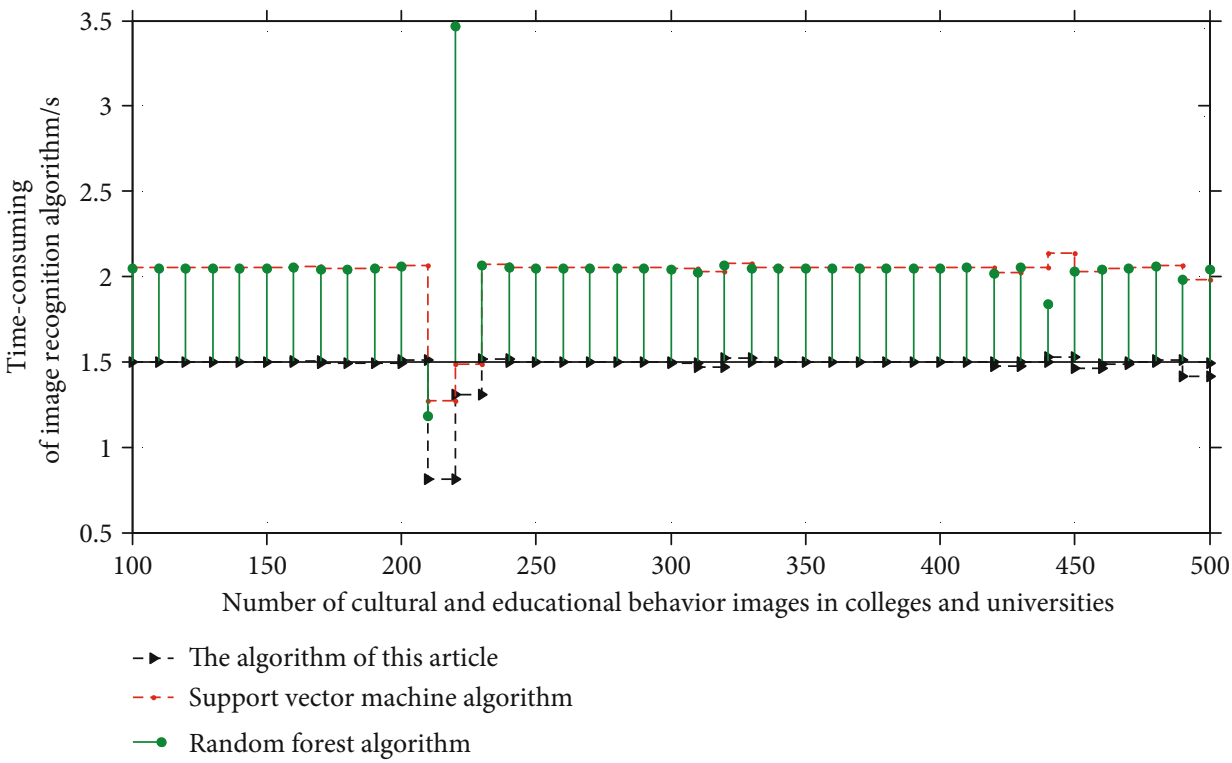

FIgURE 8: Time-consuming comparison of different image recognition algorithms.

accuracy of different image recognition algorithms is shown in Figure 7. It can be seen that the multiobjective SVM based on three decision-making theories in this paper has higher accuracy in image recognition of cultural and educational behaviors in colleges and universities.

Student satisfaction has a significant direct positive effect on students' behavior tendency. Service quality has a direct positive impact on perceived value and student trust. The direct impact of service quality on student satisfaction is not significant, but it has an indirect impact on student satisfaction through perceived value and student trust and has an indirect impact on student satisfaction through student trust and student satisfaction. The image of colleges and universities acts on student satisfaction through perceived value and student trust. The image of colleges and universities has a direct impact on student behavior tendency, and it also acts on student behavior tendency through student trust and student satisfaction. Among the influences on behavior tendency, the direct positive influence of student trust is the biggest, followed by the image of colleges and universities and the quality of service. In addition, the timeconsuming comparison of different image recognition algorithms is shown in Figure 8. It can be seen that the time consumption of the multiobjective SVM image recognition of university cultural education behavior based on three decision-making theories in this paper is the lowest, which 
shows that the real-time performance of the algorithm in this paper is the best.

\section{Conclusion}

This paper introduces three decision-making ideas when improving traditional image recognition algorithms for cultural and educational behaviors in colleges and universities. Through theoretical analysis, it is concluded that the threebranch decision can be applied to the field of cultural and educational behavior image recognition in colleges and universities, and then, the traditional college cultural and educational behavior image classification algorithm is improved, from the traditional two-branch division to the threebranch decision. The process is more in line with the way of human thinking. A classifier for image recognition of cultural and educational behaviors in colleges and universities based on three decision-making theories is designed and constructed. Experiments have proven that the feasibility and accuracy of the classifier have solved the combination of three decision-making theories and the field of cultural and educational behavior image recognition in colleges and universities. In view of the problem that the traditional image segmentation of cultural and educational behaviors in colleges and universities are all regular rectangles and the same subobject may be divided into different submodules, the three-branch decision-based image recognition classifier for cultural and educational behavior in colleges and universities proposed in this paper proposes the idea of delayed decision-making. The selection of judgment conditions is the retraining of the results of the previous classification, through a limited number of iteration which makes the image segmentation of college cultural and educational behavior images segment the same target into the same submodule, thereby reducing the error generated by the image segmentation of cultural and educational behavior in colleges and universities. The recognition error may be caused by the image segmentation of the cultural and educational behaviors in the traditional university cultural and educational behavior image recognition, so as to improve the accuracy of the cultural and educational behavior image recognition in colleges and universities. The empirical results show that service quality has a direct and positive impact on perceived value and student trust, and the direct impact of service quality on student satisfaction is not significant. The image of colleges and universities acts on student satisfaction through perceived value and student trust. The image of colleges and universities has a direct impact on student behavior tendency, and it also acts on student behavior tendency through student trust and student satisfaction. Among the influences on behavior tendency, the direct positive influence of student trust is the biggest, followed by the image of colleges and universities and the quality of service. Interactivity has an important influence on students' perception of education services. The one-factor analysis of variance in the impact of teacher-student relationship and classmate relationship on perceived education services shows that students with good teacher-student relationship perceive education services higher and students with good classmate relationships perceive education services higher.

\section{Data Availability}

The data used to support the findings of this study are included within the article.

\section{Conflicts of Interest}

All the authors do not have any possible conflicts of interest.

\section{Acknowledgments}

This study received funding from the general projects of the National Social Science Fund "Study on industrialization and urban development in northeast China (1949-1957)" (item number 21BZS0161) in 2021; the General Project of Social Science Fund of Liaoning Province (Special Project of Ideological and Political Science) "Research on the integration of anti-Japanese culture into ideological and political education in universities, middle schools and primary schools in Liaoning Province" (item number L20BSZ057) in 2020; the Ministry of Education Cooperation Education Project (teaching content and curriculum system reform project) (2nd batch of 2019) “People's Digital Horse Hospital," Beijing Culture Online (item number 22); the ideological and political education integration construction of universities, middle schools, and primary schools in Liaoning Province (antiunion spirit) (item number 202101011); and 2020 Education Department Scientific Research Topic "Liaoning Red Tourism Education function realization research" (W2020lkyjc-01).

\section{References}

[1] M. Andrejevic and N. Selwyn, "Facial recognition technology in schools: critical questions and concerns," Learning, Media and Technology, vol. 45, no. 2, pp. 115-128, 2020.

[2] M. White, R. Berry, and R. F. Rodgers, "Body image and body change behaviors associated with orthorexia symptoms in males," Body Image, vol. 34, pp. 46-50, 2020.

[3] C. Longobardi, M. A. Fabris, L. E. Prino, and M. Settanni, "The role of body image concerns in online sexual victimization among female adolescents: the mediating effect of risky online behaviors," Journal of Child \& Adolescent Trauma, vol. 14, no. 1, pp. 51-60, 2021.

[4] P. Foroudi, A. Nazarian, S. Ziyadin et al., "Co-creating brand image and reputation through stakeholder's social network," Journal of Business Research, vol. 114, pp. 42-59, 2020.

[5] E. O. McGee, "Interrogating structural racism in STEM higher education," Educational Researcher, vol. 49, no. 9, pp. 633644, 2020.

[6] A. Yaghi and R. Bates, "The role of supervisor and peer support in training transfer in institutions of higher education," International Journal of Training and Development, vol. 24, no. 2, pp. 89-104, 2020.

[7] G. Yi, "From green entrepreneurial intentions to green entrepreneurial behaviors: the role of university entrepreneurial support and external institutional support," International Entrepreneurship and Management Journal, vol. 17, no. 2, pp. 963-979, 2021. 
[8] D. Mungra, A. Agrawal, P. Sharma, S. Tanwar, and M. S. Obaidat, "PRATIT: a CNN-based emotion recognition system using histogram equalization and data augmentation," Multimedia Tools and Applications, vol. 79, no. 3-4, pp. 2285-2307, 2020.

[9] I. Korolyov and L. Domylivska, "Mentality and national character as semiotic regulators of communicative behavior," Journal of History Culture and Art Research, vol. 9, no. 1, pp. 354-364, 2020.

[10] M. Azam and J. A. Qureshi, "Building employer brand image for accumulating intellectual Capital," Estudios De Economia Aplicada, vol. 39, no. 2, 2021.

[11] L. Anthonysamy, A. C. Koo, and S. H. Hew, "Self-regulated learning strategies in higher education: fostering digital literacy for sustainable lifelong learning," Education and Information Technologies, vol. 25, no. 4, pp. 2393-2414, 2020.

[12] F. A. Yusuf, "The effect of organizational culture on lecturers' organizational commitment in private universities in Indonesia," International Journal of Higher Education, vol. 9, no. 2, pp. 16-24, 2019.

[13] D. Schubbe, P. Scalia, R. W. Yen et al., "Using pictures to convey health information: a systematic review and meta-analysis of the effects on patient and consumer health behaviors and outcomes," Patient Education and Counseling, vol. 103, no. 10, pp. 1935-1960, 2020.

[14] N. Saenko, O. Voronkova, E. Zatsarinnaya, and M. Mikhailova, "Philosophical and cultural foundations of the concept of "nihitogenesis"," Journal of Social Studies Education Research, vol. 11, no. 1, pp. 88-103, 2020.

[15] J. Sheth, "Impact of Covid-19 on consumer behavior: will the old habits return or die?," Journal of Business Research, vol. 117, pp. 280-283, 2020.

[16] A. Bibi, A. Saleem, M. A. Khalid, and N. Shafique, "Emotional intelligence and aggression among university students of Pakistan: a correlational study," Journal of Aggression, Maltreatment \& Trauma, vol. 29, no. 10, pp. 1189-1203, 2020.

[17] K. Cheung, K. Y. Tam, M. H. Tsang, L. W. Zhang, and S. W. Lit, "Depression, anxiety and stress in different subgroups of first-year university students from 4-year cohort data," Journal of Affective Disorders, vol. 274, pp. 305-314, 2020.

[18] K. Brundiers, M. Barth, G. Cebrián et al., "Key competencies in sustainability in higher education-toward an agreed-upon reference framework," Sustainability Science, vol. 16, no. 1, pp. 13-29, 2021.

[19] P. Parvathy, K. Subramaniam, G. K. D. Prasanna Venkatesan, P. Karthikaikumar, J. Varghese, and T. Jayasankar, "Development of hand gesture recognition system using machine learning," Journal of Ambient Intelligence and Humanized Computing, vol. 12, no. 6, pp. 6793-6800, 2021.

[20] J. Sukhera, C. J. Watling, and C. M. Gonzalez, "Implicit bias in health professions: from recognition to transformation," Academic Medicine, vol. 95, no. 5, pp. 717-723, 2020. 